\title{
Breaking bad news in genetic counseling-problems and communication tools
}

\author{
Magdalena M. Witt ${ }^{1} \cdot$ Katarzyna A. Jankowska ${ }^{2}$
}

Received: 22 August 2018 / Revised: 11 September 2018 / Accepted: 13 September 2018 / Published online: 25 September 2018

(C) The Author(s) 2018

\begin{abstract}
Breaking bad news is a common problem for clinical geneticists in their daily work. Just like doctors of other specialties, e.g., oncologists, they can use proven communication tools instead of relying only on professional sense. The latter is, of course, always the most important for experienced doctors, but the use of protocols such as SPIKES and EMPATHY facilitates both the delineation of difficult information and the process of its transmission. The article gives an overview of the best tools of this type available to medical professionals dealing with genetic counseling.
\end{abstract}

Keywords Breaking bad news · Communication skills · EMPATHY · Genetic counseling · SPIKES

The issue of exceptionalism of genetic data (a concept that genetic information is qualitatively unique from other medical data of general purpose and therefore raises unique social, psychological, legal, and ethical issues) has been debated over decades (Witt and Witt 2016). Currently, since it is acknowledged that a hereditary component can be involved in numerous chronic disorders, e.g., heart disease, cancer, and dementia, the classical genetic disorders are not the only object of interest of clinical genetics. Thus, a need for competency in genomic medicine has been widened to many other medical specialties (Kumar and Eng 2015). Numerous aspects of genetic information are collected in the process of diagnosing genetic disorders or genetic components of chronic diseases (mainly pediatric). They cause severe consequences of personal, familial, and social nature that markedly distinguish genetic disorders/components from nonhereditary medical

Communicated by: Michal Witt

Magdalena M. Witt

magdalenawitt@wp.pl

1 Department of Medical Rescue, Poznań University of Medical Sciences, Dąbrowskiego 79, 60-529 Poznań, Poland

2 Department of Pediatrics, Hematology and Oncology, The Collegium Medicum of the Nicolaus Copernicus University, Bydgoszcz, Poland conditions (Bennett 2010). The genetic traits causing pathologies are distinctive in several aspects.

1. Due to the fact of being familial, the genetic diagnosis may deeply affect everyday life of many individuals. This influence may extend far beyond a nuclear family particularly in case of dominant or X-linked inheritance. Typical manifestations of this include parental guilt following indication that genetic factors are transmitted to the next generation; threatening of familial confidentiality and intrusion of privacy due to the fact that in genetic counseling extended family is a care unit; altering of reproductive plans because of the knowledge of genetic risk factors, even by distant relatives; threatening of individuals' notion of presumed parental roles; and challenges to religious and ethical beliefs.

2. Genetic traits are permanent in nature - therapeutic approaches are still deeply in their infancy and their prospects to become a reality in a larger scale have no rational justification. This frequently causes deep frustration that the individual cannot alter the genetic heritage, and further real destiny in life.

3. Genetic traits are mostly chronic and frequently progressive. Many individuals become increasingly impaired by their condition with age. This becomes the cause of a growing care problem, creating continual strains on the patient and/or family.

4. Genetic traits are complex and usually classified as rare diseases. Patients and their families constantly fight to 
find the right medical center, a competent specialist, and the appropriate path of medical intervention.

5. Stigmatization is a common consequence of the disclosure of phenotype and/or genetic heritage. Genetic label can cause perceiving himself/herself different and flawed, which results in developing succeeding identity problems and grieve. Stigmatization entails discrimination in many areas of life: familial, social, psychological, religious, etc.

6. A sense of threat and insecurity is frequent among genetic patients/families. This affects any aspect of life, from reproductive decisions to self-esteem, lifestyle, and even longevity.

7. Probabilistic results of genetic testing are frequently accompanied by uncertainty, particularly for susceptibility testing; negative results of presymptomatic testing can cause survivor guilt, while positive almost always lead to a depressive conduct.

An awareness of serious psychosocial consequences must always accompany the whole process of genetic counseling (Harper 2001). The ability to mitigate these negative effects is an important element of providing counseling in a professional mode. A smooth transfer of the whole, often very difficult in perception, complex knowledge depends greatly on the communication skills of a counselor.

Genetic counseling has the information and education dimension. However, because of serious consequences for the life of the patient/patient's family, it must also have a deep human dimension. The problem extends far beyond, e.g., parents who have received the results of a genetic test on their children face a dilemma of how and when to inform them. According to a large-scale systemic review, the biomedical and educational approach dominates the counseling process, while consideration of the psychological aspects needed during such a dialogue is absent (Paul et al. 2015). This indicates that preparation of medical geneticists for breaking bad news is crucial for an effective achievement of the human aspect of genetic counseling.

Information on the results of genetic tests in the vast majority of cases falls within the classic definition of bad news, which is any news that will adversely and seriously affect one's future" (Baile et al. 2000; Lloyd and Bor 2009). Effective communication of the bad news is not an easy task due to an uncertainty about the patient's expectation, fear of destroying the patient's hope, fear of counselor's own inadequacy in the face of uncontrollable disease, and/or not feeling prepared to manage anticipated emotional reactions of the patient (Baile et al. 2000).

A proper approach to the problem of breaking bad news aims to familiarize the patient/family with the message at the verbal (intellectual) level while taking care of the emotional state of the counselee during consultation. It should also involve the patient in making decisions and offer realistic hope for the future regardless of severity of medical problem according to the principle that in medicine one can and should always offer something reasonable to a patient (there is always something beneficial to be done).

The elements necessary to take into account, which may be helpful during the preparation for submitting the bad news, are catalogued below:

- Does the patient expect bad news?

- Who else should be present during such a conversation (family member, other representative of the medical staff)?

- What has the patient been told or have read about the disease so far?

- Does the meeting take place in appropriate conditions (place, time intended, etc.)?

- What is the previous experience related to the genetic diagnostic process of the patient?

- What are social conditions of the patient?

The process of transmitting bad news can be summarized as follows (Lloyd and Bor 2009):

Give information

$\downarrow$

Check the patient's understanding of the information

$\downarrow$

Identify the patient's main concern

$\downarrow$

Elicit the patient's coping strategies and personal resources $\downarrow$

Give realistic hope

In order to facilitate the difficult conversation between the physician involved in presenting unfavorable results/ prognosis and the patient, the SPIKES protocol was developed based on Buckman's model of breaking bad news, originating and used on a large scale mainly in oncology (Baile et al. 2000). It builds on the strategy of six consecutive steps and creates settings to conduct the conversation in a compassionate, caring, and informative way (Baile et al. 2000; Daly et al. 2001). It is intended for conducting any medical consultation, during which it comes to disclosing unfavorable medical information to a patient, and is perfectly suited for use in genetic counseling. Its component parts corresponding to the acronym are:

- $\quad \mathrm{S}$-Setting

- P-Perception

- I-Invitation

- $\mathrm{K}$-Knowledge

- E-Emotions, Empathy

- S-Strategy, Summary

Setting-Refers to the conditions of the place where the meeting takes place, but also the way of building interpersonal 
relation between the doctor and the patient; it is also a decision on whether there is a need for the presence of other people like family members, another doctor, psychologist, and nurse.

Perception - Pertains to the state of the patient's awareness of his illness based on the previously obtained information (contact with another doctor, the Internet).

Invitation - Gives the patient a choice regarding the scope of information provided (general context, details).

Knowledge-A way of passing information communicatively, in parts, using pauses and repetitions, which allows the counselee to deal with both the facts and the accompanying emotions.

Emotions, empathy - Understanding, kindness, and support shown throughout the conversation.

Strategy, summary - A closing summary with an outline of the action plan for the future, additional questions preferably given also in writing (Baile et al. 2000).

Another tool that can be helpful during difficult conversations in the physician's office is the EMPATHY protocol containing principles essential for proper transmission of unsuccessful results of diagnostic tests (Jankowska 2014). A specific application of the EMPATHY protocol is to inform parents on the results of their child's genetic tests during the genetic counseling process. It has been documented that parental experiences are more positive when geneticists informing about the diagnosis are not verbally dominant, provide enough emotional support, less frequently use medical jargon, give hope, and explain plans for further action (Ashtiani et al. 2014). It is also known that the first experiences parents have from interviews with a geneticist can have a significant and long-term impact on coping with the care of a sick child (Waxler et al. 2013). The counselor giving unfavorable messages to parents of the affected child has to fulfill the double obligation, combining knowledge and technical skills with the understanding of the needs of the whole family (Davies et al. 2003).

EMPATHY is an acronym of the protocol which has a goal of the cognitive binding of the process of passing on bad information with empathy; proper conduct empathy is understood as perceiving the emotions of the patient/parents and understanding them.

\section{E-Emotions}

Both in the context of reporting research results and demonstrating emotional support needed by the patient.

\section{M-Meeting}

For the quality of the meeting with the patient, its place is important, not only in the physical sense but also regarding the absence of any disturbing factors and the time intended for it.

\section{P-Patient's perspective}

Hearing even a few introductory sentences of the patient on the subject allows for a more effective conversation, by creating more possibilities to adapt it to the counselee competence.

\section{A-Adequate language}

Using a language that is unambiguous and understandable for the person to whom the results of genetic testing are transmitted; any deviations from this rule result in lower compliance and an increase in anxiety in patients.

T and H-Truth and hope - two letters, one phone

Respecting the patient's right to the truth about his illness, but this truth should be conveyed in a way that does not take away hope (Roshanai et al. 2009).

\section{Y-Yes for patient empowerment}

The purpose of the proper form of conversation is to maintain the patient's autonomy, build his sense of influence, faith in his own strength, and responsibility for the course of treatment.

\section{Conclusion}

It is widely believed that good communication is the core of good medical care. The method of medical consultation affects not only the doctor's relationship with the patient, which underlies mutual trust, but also whether the patient will follow the instructions of the consultant, perform further diagnostic tests, make efforts to change the habits of life, inform extended family, etc. A good relationship also reduces stress and burnout in medical staff, reduces the number of complaints and lawsuits, and underlies satisfaction with daily work. These statements refer in a special way to clinical genetics. The purpose of genetic counseling is to provide highly sophisticated and value-laden knowledge of complex and often abstract information that may influence the overall functioning of the patient and his family. Therefore, it has to be conveyed in a way allowing the data received to be helpful in understanding and assimilating the adaptation to situation, or the risks that it brings (Paul et al. 2015). The specific nature of genetic counseling requires a particular delicacy and sensitivity from a counselee. Therefore, application of proven communication tools, based on the scientifically justified methods, is particularly desirable.

There is a strong scientific evidence that clinical communication as a skill can be taught and retained (Baile et al. 2000). The methods of successful communication should be 
taught to students and medical professionals involved in genetic counseling. Among the various teaching methods, the most recommended are programs combining the transfer of a theoretical knowledge with practical rehearsals and constructive feedback, distributed over the years of academic education (Gysels et al. 2005). Teaching communication at a medical university should be obligatory, divided into stages and taught based on the international standards, using recognized and accepted protocols like the ones presented above.

\section{Compliance with ethical standards}

Conflict of interest The authors declare that they have no conflict of interest.

Ethical approval This article does not contain any studies with human participants or animals performed by any of the authors.

Open Access This article is distributed under the terms of the Creative Commons Attribution 4.0 International License (http:// creativecommons.org/licenses/by/4.0/), which permits unrestricted use, distribution, and reproduction in any medium, provided you give appropriate credit to the original author(s) and the source, provide a link to the Creative Commons license, and indicate if changes were made.

\section{References}

Ashtiani S, Makela N, Carrion P, Austin J (2014) Parents experiences of receiving their child's genetic diagnosis: a qualitative study to inform clinical genetics practice. Am J Med Genet 164A:1496-1502
Baile WF, Buckman R, Lenzi R, Glober G, Beale EA, Kudelka AP (2000) SPIKES -a six-step protocol for delivering bad news: application to the patient with cancer. Oncologist 5:302-311

Bennett RL (2010) The practical guide to the genetic family history. Wiley-Blackwell, Hoboken

Daly MB, Barsevick A, Miller SM, Buckman R, Costalas J, Montgomery S, Bingler R (2001) Communicating genetic tests results to the family a sixth-step skills-building strategy. Fam Community Health 24(3):13-26

Davies R, Davis B, Sibert J (2003) Parents stories of sensitive and insensitive care by paediatricians in the time leading up to and including diagnostic disclosure of a life-limiting condition in their child. Child Care Health Dev 29:77-82

Gysels M, Richardson A, Higginson IJ (2005) Communication training for health professionals who care for patients with cancer: a systemic review of training methods. Support Care Cancer 13:356-366

Harper PS (2001) Practical genetic counselling, 5th edn. Arnold, London

Jankowska A (2014) Medical communication in pediatric oncology. Passing information on diagnosis of cancer (in Polish). Psychoonkologia 4:123-127

Kumar D, Eng C (2015) Genomic medicine. Principles and practice, $2^{\text {nd }}$ edition. Oxford University Press, Oxford

Lloyd M, Bor R (2009) Communication skills for medicine. Elsevier, New York

Paul J, Metcalfe S, Stirling L, Wilson B, Hodgson J (2015) Analysing communication in genetic consultation - a systemic review. Patient Educ Couns 98:15-33

Roshanai A, Rosenquist R, Lampic C, Nordin K (2009) Does enhanced information at cancer genetic counseling improve counselees' knowledge, risk perception, satisfaction and negotiation of information to at-risk relatives? - a randomized study. Acta Oncol 48:9991009

Waxler JL, Cherniske EM, Dieter K, Herd P, Pober BR (2013) Hearing from parents: the impact of receiving the diagnosis of Williams syndrome in their child. Am J Med Genet 161:534-541

Witt MM, Witt MP (2016) Privacy and confidentiality measures in genetic testing and counselling: arguing on genetic exceptionalism again? J Appl Genet 57:483-485 